\title{
THREE TYPES OF LINACS FOR CUSTOMS LARGE CONTAINER INSPECTION APPLICATION*
}

\author{
Accelerator Lab ${ }^{\dagger}$, Tsinghua University, Beijing, 100084, P.R.China \\ Email: LYZ@dns.ep.tsinghua.edu.cn
}

\begin{abstract}
In recent years, three types of linacs have been developed for large container inspection for customs application, viz., S-Band 9MeV travelling-wave (TW) linacs, S-Band 6MeV Standing-wave (SW) linacs and XBand $2.5 \mathrm{MeV}(\mathrm{SW})$ linacs. In cooperation with Tsinghua Tongfang Co. Ltd, a series of container inspection systems has been produced. Seven sets of large container inspection systems with $9 \mathrm{MeV}$ TW linacs have been installed at Tianjin, Dalian, Qingdao, Shanghai, Dapeng, and Nanjing Seaport customs. These systems are called "the fixed type". Based on our development of a compact $\mathrm{X}$-band $9300 \mathrm{MHz} 2.5 \mathrm{MeV}$ SW linac, a novel "mobile type" large container inspection system was completed. Four sets of these systems have been installed in four customs areas. Another container inspection system with a S-Band $6 \mathrm{MeV} \mathrm{SW}$ linac, installed in a simple shielding shed with cement blocks, is called "the combined type". Four sets of these systems have been equipped in four customs areas.
\end{abstract}

\section{INTRODUCTION}

Smuggling in China continues to be a serious problem. The smuggling of contraband goods, arms and drugs, as well as import duty avoidance is not only an important security problem, but also affects the national income in term of import and export duty. These issues are a serious concern to the government. Usually, container inspection methods rely on manual procedures, which necessitate only a few percent of containers being inspected. This is no longer acceptable with increasing numbers of containers being transported. An automated inspection system is necessary, such as that found in airports for inspecting personal luggage. The Chinese government has made a decision to equip customs with advanced devices. To meet these requirements, three types of large container inspection systems for customs have been developed by Tsinghua University in cooperation with Tsinghua Tongfang Co. Ltd.

The principle of these systems is as follows: Highenergy X-rays emerging from a linear accelerator scan the container. The detectors acquire the penetrated radiation through the container. The electrical signals converted by the detectors are amplified and digitized. Finally, the X-ray image of the penetrated container is displayed on a video monitor at the desk of the customs

\footnotetext{
* Supported by the National Natural Science Foundation and National Science and Technology Program.

${ }^{\dagger}$ Presented by Lin, Yuzheng.
}

officer. The system provides a fast inspection procedure for the goods without the necessity of opening the container.

Three new types of linear accelerators, S-band $9 \mathrm{MeV}$ travelling-wave(TW) linacs, X-band $2.5 \mathrm{MW}$ standingwave (SW) linacs and S-band 6.0MW SW linacs have been developed for large container inspection systems. In cooperation with Tsinghua Tongfang Co. Ltd, a series of container inspection systems has been mass-produced. About fifteen sets of the systems have been installed at seaports and other ports. This paper gives an overview of these three types of linacs and their applications for customs.

\section{S-BAND 9MEV LINACS FOR THE FIXED TYPE SYSTEMS}

More than ten seaports around the Chinese seacoast need to be equipped with the above container systems. At these seaports, more than 1000 containers are transported every day, which cannot be all inspected by manual methods. A system needs to be developed to rapidly inspect the containers using very strong $\mathrm{X}$-rays to penetrate the heavy containers. A $9 \mathrm{MeV}$ linac with a dose rate exceeding $4000 \mathrm{cGy} / \mathrm{min} . \mathrm{m}$ as the radiation source meets the requirement. Given a requirement for operational stability, but no serious space limitation, a travelling wave (TW) accelerating type was selected. With extensive space for installing the system in seaports, we adopted a kind of platform car system on the iron tracks which rapidly move the container for checking purposes. The $9 \mathrm{MeV}$ TW linac is situated in a shielded room, leaving a thin vertical slit to let the X-rays through. The X-rays going out from the shielded room is collimated first by a thin lead slit. Another collimation lead slit is placed in front of a linear detector array, opposite the linac. The platform car with the inspected container moves through the area between the first and second collimators, ensuring that the X-rays scan the total container from "head" to "tail".

The $2 \pi / 3$ mode $9 \mathrm{MeV}$ linac is driven by a $3.5 \mathrm{MW}$ klystron, which has a $0.4 \mathrm{~m}$ long bunching section and a $1.8 \mathrm{~m}$ long main accelerating waveguide. The beam pulse current is about $120 \mathrm{~mA}$, and pulse length $5 \mu \mathrm{s}$. The bunching capture efficiency is more than $75 \%$. The electron beam emerging from a Pierce electron gun is focused and steered, then enters the bunching section. Another set of main solenoid coils serves to focus the electron beam. In order to further focus the accelerated beam to $\phi 1.5 \mathrm{~mm}$, a triple-quadrupole lens is employed in the front of target. A special $3.5 \mathrm{~mm}$ tungsten target structure can remove the $1.1 \mathrm{KW}$ beam heat load which 
can be centralized to a size of $\phi 1.5 \mathrm{~mm}$. The dose rate is more than $4000 \mathrm{cGy} / \mathrm{min} . \mathrm{m}$ with duty rate $250 \mathrm{pps}$. Fig. 1 shows the view of structure of the $9 \mathrm{MeV}$ TW linac from the electron gun side.

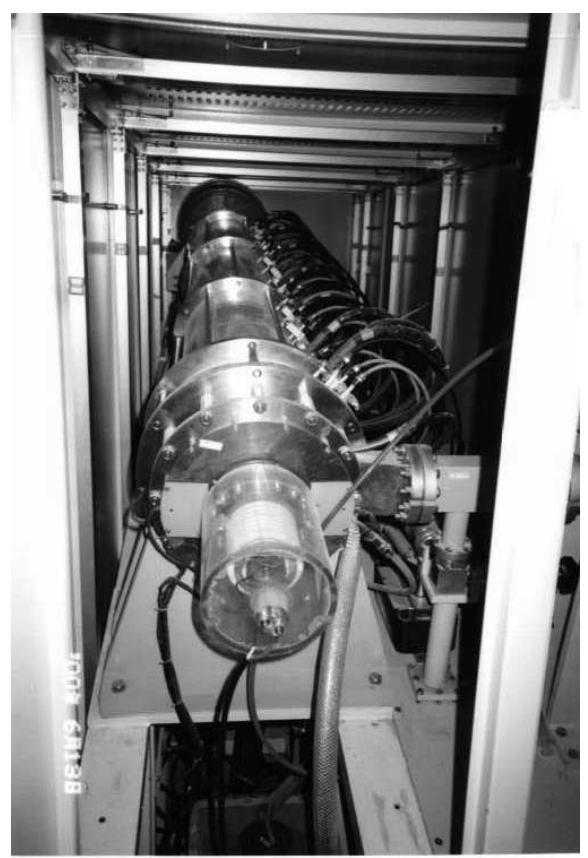

Figure 1: View of structure of the $9 \mathrm{MeV}$ TW linac from the electron gun side.

During the tuning and matching of the input and output coupler, a modified technique is developed ${ }^{[1]}$. Using this technique, the frequency difference $\Delta f$ and coupler coefficient $\beta$ can be calculated by the measured results of reflecting parameters with a vector analyzer.

The radiation head of the linac is shown as Fig.2. The microwave power source is situated in other room.

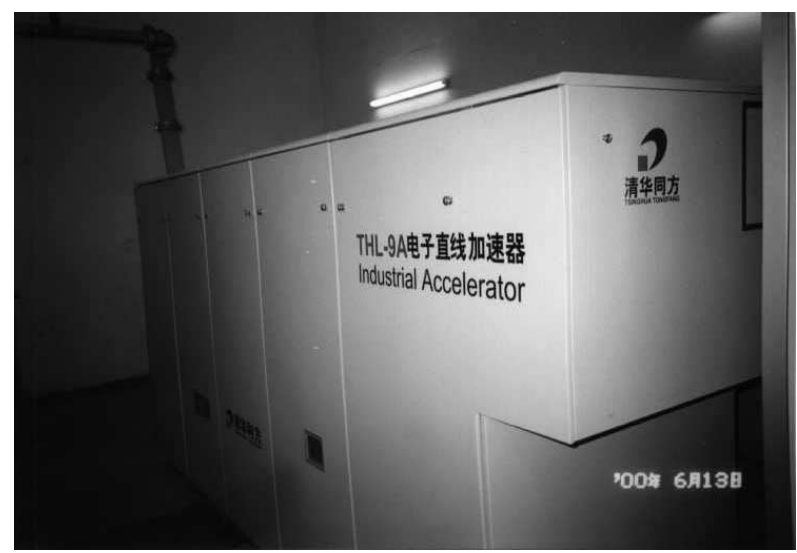

Figure 2: View of the radiation head of the linac.

Seven sets of these systems have been installed at the seaport customs areas in Tianjin, Dalian, Qingdao, Shanghai etc. Fig. 3 shows the building and working field of the system at Tianjin seaport customs.

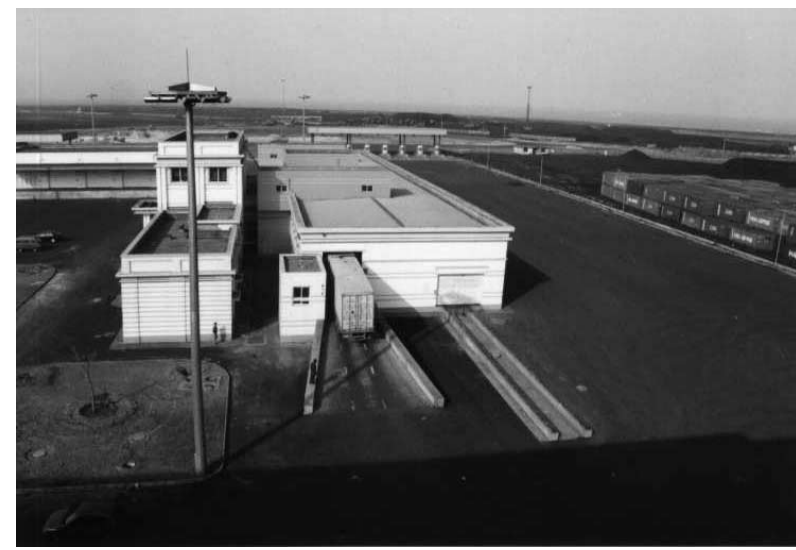

Figure 3: View of the building and working field of the system at Tianjin seaport customs.

\section{X-BAND 2.5MEV SW LINACS FOR THE MOBILE TYPE SYSTEMS}

While most ports have insufficient space to install the above mentioned "fixed type" inspection system. They also have less containers to inspect. In this case, a kind of mobile, flexible, self-shielding, compact system is necessary. In order to meet these requirements, a new system has been developed, which is mounted in a truck. In the truck, a radiation head, including accelerating tube, modulator, magnetron, cooling system etc, is situated in one corner. The control room can be put above the truck, or set up inside a separate truck. Sometimes a diesel generating set is required as a power source. A $\Gamma$ type detector array is mounted at a movable "arm", which can be put back on the truck when the truck is moved. System mounted in a truck should be very compact and low power consumption. Fig.4 provides a picture of the mobile container inspection system.

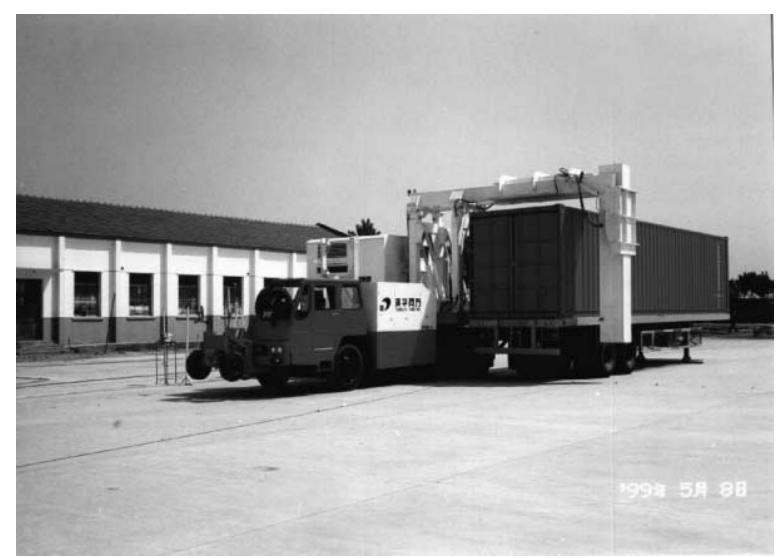

Figure 4: View of the mobile container inspection system.

In the system, an X-band $2.5 \mathrm{MeV}$, on-axis coupled standing-wave linac is selected as a radiation source, operated in a $\pi / 2$ mode. The length of the accelerating guide is only $15 \mathrm{~cm}$, with a diameter of less than $4 \mathrm{~cm}$. The accelerating guide consists of 21 accelerating Cells. To minimize the size and weight of the structure and to improve the beam spot as well as transportation, a phasefocusing technique is used without any external- 
magnetic focusing solenoid coils. By choosing the right phase velocity distribution and tapering the field magnitude of the buncher, longitudinal bunching and acceleration and transverse focusing can be achieved simultaneously. The microwave power source is a 1.0MW tunable coaxial magnetron at $9.3 \mathrm{GHz}$, developed by the Beijing Institute of Electronic \&Vacuum Technology (BIEVT).

A very important feature of the system is selfshielding. The X-rays are almost completely shielded by a very heavy tungsten shell except for a thin flat vertical slit, which allows the X-rays through. The attenuation efficiency of the X-ray can reach $10^{-5}$. So the system is very safe. Customs officers can operate it by the side, or even inside the truck. Fig.5 shows the complex of its radiation head of the system.

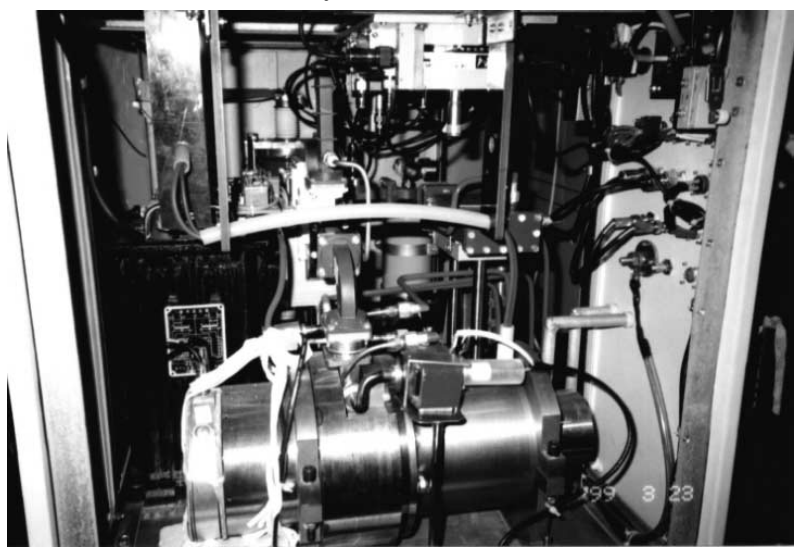

Figure 5: View of the radiation head of the mobile container inspection system.

In cooperation with Tsinghua Tongfang Co. Ltd, four sets of these systems have been produced, and placed in Xiamen and Guangzhou.

\section{S-BAND 6MEV SW LINACS FOR THE COMBINED TYPE SYSTEMS.}

For middle scale ports with an average number of transported containers of 300 500 per day, the "fixed type" system is considered rather expensive and the "mobile type" system is inadequate. In these cases, middle scale system is required which can be placed in a simply-shielded cement-block building but possessing more stronger penetration power than the mobile one. To meet this requirement, a "combined type" inspection system has been developed also in cooperation with Tsinghua Tongfang Co. Ltd.

A double-housing gantry structure is employed in this system (Fig.6), with the radiation head mounted on one side of the gantry. The detector array is put in the other side and on top of the gantry, forming a $\Gamma$ type structure. The modulator and the cooling system are placed on the opposite side to the radiation head. The primary tungsten collimator and front collimator are placed at the exit of the radiation head, with the back collimator being set at the entry of the detector array.

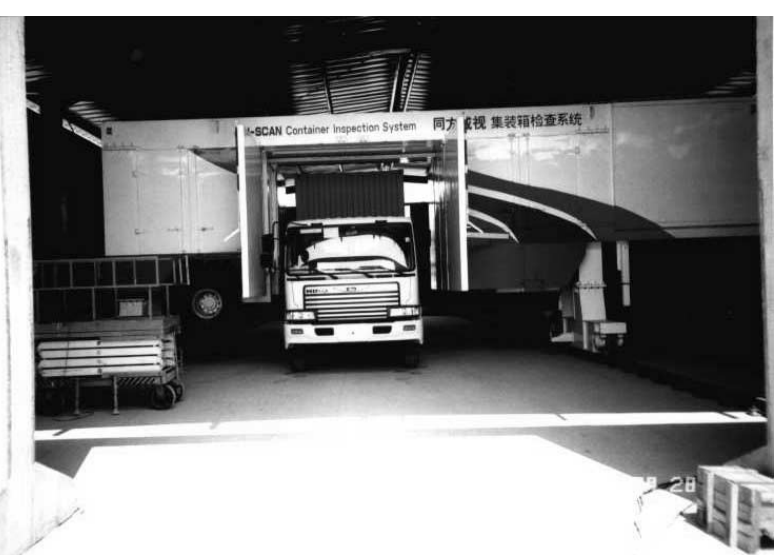

Figure 6: View of the double-housing gantry structure.

In this system, it is necessary to increase the X-ray energy. A S-band $6.0 \mathrm{MeV}$ standing-wave linac is a suitable choice for a radiation source. The X-rays can penetrate the container with the equivalent of $250 \mathrm{~mm}$ thick iron-plate. The length of the accelerating tube with an on-axis coupler is about $28 \mathrm{~cm}$, consisting of six accelerating cavities, including one buncher cavity. The pulse beam current is about $100 \mathrm{~mA}$. The linac is driven by a 2.6MW Marconi Company (EEV) tunable magnetron at $2998 \mathrm{MHz}$, with pulse width being $4.2 \mu \mathrm{sec}$.

In this combined-type system, self-shielding is still an important issue, as the cement-block wall is not so thick and is insufficient to shield the X-ray. The attenuation factor of the tungsten shell should be $10^{-4}$ at one meter distance. Fig.7 shows the radiation head in which the accelerating structure, tungsten shielding body, pulse transformer, four-port circulator, etc, are mounted.

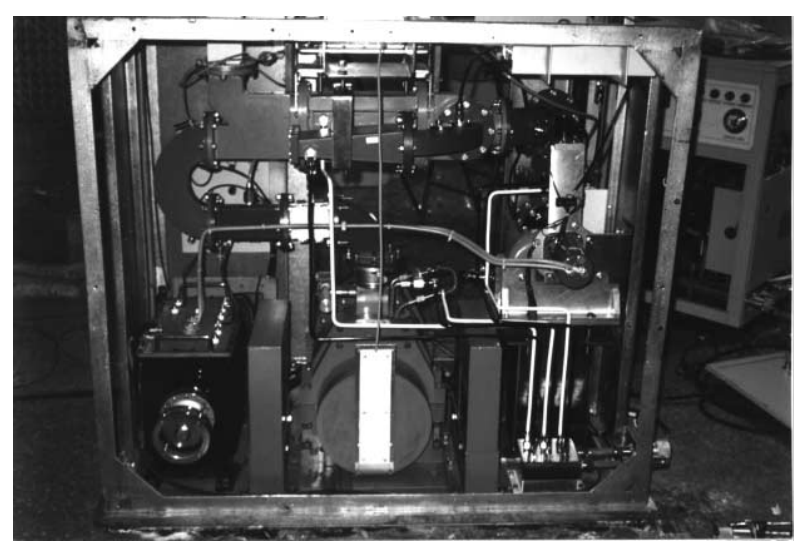

Figure 7: View of the complex of radiation head.

To date, four sets of these systems have been installed in Beijing, Xiamen, Shanghai, and Guangzhou customs areas.

\section{REFERENCES}

[1] S. X. Zheng et al, "A Quantitive Method of Coupler Cavity Tuning and Simulation", these proceedings. 